\title{
Phase II trial of granulocyte-macrophage colony-stimulating factor plus thalidomide in older patients with castration-resistant prostate cancer
}

\author{
LEI SONG, XIJIAN ZHOU and XIANGYONG LI \\ Department of Hematology and Oncology, The 101th Hospital of The People's \\ Liberation Army, Wuxi, Jiangsu 214044, P.R. China
}

Received January 20, 2015; Accepted April 24, 2015

DOI: $10.3892 / \mathrm{mco} .2015 .571$

\begin{abstract}
The objective of this study was to assess the efficacy of granulocyte-macrophage colony-stimulating factor (GM-CSF) in combination with thalidomide for prostate-specific antigen (PSA) reduction in older patients (aged $\geq 70$ years, life expectancy of $>1$ year) with castration-resistant prostate cancer (CRPC). A total of 11 CRPC patients were treated with $300 \mu \mathrm{g}$ GM-CSF administered subcutaneously on days 1, 3 and 6 of weeks 1 and 2 of each cycle. Thalidomide was gradually increased to reach the study dose of $100 \mathrm{mg} /$ day. The patients were assessed every 4 weeks with therapy continuing to 4 months. All 11 patients exhibited a decrease in PSA levels and 3 patients (27.2\%) exhibited a PSA decrease of $>50 \%$ in cycle 1 . In cycle 2,8 patients exhibited decreasing PSA levels. A total of 3 patients (27.2\%) had a PSA rebound, with 1 patient exhibiting a PSA rebound of $>50 \%$. In cycle 3,10 patients exhibited continuously decreasing PSA levels, with 2 patients (18.2\%) exhibiting a PSA decrease of $>50 \%$; 1 patient $(27.2 \%)$ had a PSA rebound of $<50 \%$. In cycle 4,9 patients exhibited continuously decreasing PSA levels and 2 patients (18.2\%) had a PSA rebound of $>50 \%$. All 11 patients in this study exhibited a decrease in PSA levels, with a median decrease of $92.2 \%$. Therapy was well tolerated, with the majority of the patients experiencing only one adverse event. In conclusion, the combination of GM-CSF with thalidomide was found to be clinically effective and well tolerated by elderly CRPC patients. Therefore, GM-CSF plus thalidomide may be considered a viable treatment option for such patients.
\end{abstract}

Correspondence to: Mr. Xiangyong Li, Department of Hematology and Oncology, The 101th Hospital of The People's Liberation Army, 101 North Xingyuan Road, Wuxi, Jiangsu 214044, P.R. China E-mail: xiangyongl@126.com

Key words: castration-resistant prostate cancer, thalidomide, granulocyte-macrophage colony-stimulating factor

\section{Introduction}

Prostate cancer is one of the most common cancers among men in China. The morbidity and mortality rates of prostate cancer were reported to be 2.74 and $2.26 \%$ in 2013; however, these rates have decreased over the last 10 years due to prostate-specific antigen (PSA) screening. The standard first-line treatment of metastatic prostate cancer is androgen-deprivation therapy (ADT) (1). However, the majority of the patients develop progressive disease in 14-30 months, which is resistant to ADT and referred to as castration-resistant prostate cancer (CRPC).

The optimal therapy for patients with CRPC is considered to be chemotherapy with taxane-based regimens, abiraterone and sipuleucel-T (2-4). However, patients aged $\geq 70$ years may not be suitable for these therapies and treatment selection should be carefully considered due to the different patient status. A phase II clinical study demonstrated that the combination of granulocyte-macrophage colony-stimulating factor (GM-CSF) and thalidomide may decrease the level of PSA and exert antitumor effects (5).

To the best of our knowledge, our study was the first to evaluate the efficacy and determine the mechanism of action of GM-CSF plus thalidomide in the treatment of CRPC patients aged $\geq 70$ years in China.

\section{Patients and methods}

Patient selection. Patients aged $\geq 70$ years with a life expectancy of $>1$ year, with histologically confirmed adenocarcinoma of the prostate, radiographic evidence of metastases, failed previous ADT and evidence of disease progression demonstrated by at least 3 consecutive increases in PSA, were considered eligible for this study. Additional requirements included an Eastern Cooperative Oncology Group (ECOG) performance status of 0 or 1 (Table I), adequate hematological function (absolute peripheral granulocyte count $\geq 1,500 / \mathrm{mm}^{3}$ and platelet count $\geq 100,000 / \mathrm{mm}^{3}$ ), adequate renal function (serum creatinine $\leq 2.0 \mathrm{mg} / \mathrm{ml}$ ) adequate hepatic function (bilirubin $\leq 1.5 \mathrm{mg} / \mathrm{dl}$ and alanine aminotransferase (ALT) $\leq 2$ times the upper limit of normal). All the patients signed an informed consent approved by the Institutional Review Board of the 101th Hospital of The People's Liberation Army, Wuxi, China. 
Treatment plan. The patients received $300 \mu \mathrm{g}$ GM-CSF subcutaneously 3 times per week (on days 1, 3 and 6 of weeks 1 and 2); thalidomide was initiated at a dose of $50 \mathrm{mg}$ at bed time, with a 50-mg increment over 5 days to reach the study dose of $100 \mathrm{mg} /$ day. One cycle was defined as 4 weeks of treament. The patients were treated continuously for 4 cycles or until disease progression, patient non-compliance or refusal to continue therapy, or development of unpredictable, irreversible, or grade 4 toxicity.

Every 4 weeks, complete blood counts, differentials and platelet counts were measured; electrolytes, blood urea nitrogen and creatinine were measured and liver function tests (aspartate aminotransferase, ALT and lactate dehydrogenase) were performed; serum PSA levels were measured on days 1 and 8 .

Considerations. The major objectives were to asses PSA response to treatment with GM-CSF plus thalidomide and to determine whether this combination is worthy of further investigation. A decrease in PSA level of $\geq 50 \%$ from the baseline was considered to be of clinical interest.

\section{Results}

Patient characteristics. A total of 11 CRPC patients were enrolled in this study. All the patients were aged $\geq 70$ years (median, 77 years), had an ECOG performance status score of 0 or 1 and had metastatic disease. The patient characteristics are summarized in Table I.

PSA response to GM-CSF plus thalidomide by treatment cycle. In cycle 1, all the patients exhibited a decrease in PSA levels, with 3 patients $(27.2 \%)$ exhibiting a PSA decrease of $>50 \%$ (Table II), which indicated that the patients responded well to this treatment. In cycle 2,8 patients exhibited continuously decreasing PSA levels. However, 3 patients $(27.2 \%)$ had a PSA rebound and in 1 patient the PSA rebound was $>50 \%$ (Table III). In cycle 3,10 patients exhibited continuously decreasing PSA levels and in 2 patients $(18.2 \%)$ the PSA decrease was $>50 \%$. In addition, 1 patient $(27.2 \%)$ had a PSA rebound, although it was $<50 \%$ (Table IV). In cycle 4, 9 patients exhibited continuously decreasing PSA levels, whereas 2 patients (18.2\%) had a PSA rebound of $>50 \%$ (Table V). All 11 patients exhibited a PSA decrease in this study, with a median decrease of $92.2 \%$ (Fig. 1).

Treatment-related toxicity. The most frequent grade 1-2 toxicities were fever, nausea, constipation, neuropathy and fatigue, whereas 1 patient developed grade 1 deep venous thrombosis (Table VI).

\section{Discussion}

The recommended therapy for patients with CRPC is chemotherapy with taxane-based regimens, abiraterone and sipuleucel-T (2-4). However, elderly patients may not be suitable for these therapies and treatment should be carefully selected. A phase II clinical study demonstrated that the combination of GM-CSF and thalidomide effectively decreased the level of PSA and exhibited antitumor activity (5).
Table I. Patient characteristics $(n=11)$.

\begin{tabular}{lr}
\hline Characteristics & Values \\
\hline Median age, years & 77 \\
Median Gleason score at diagnosis & 7 \\
ECOG performance status & 7 \\
0 & 4 \\
1 & \\
Sites of metastatic disease, no. & 10 \\
Bone & 1 \\
Extraosseous disease &
\end{tabular}

ECOG, Eastern Cooperative Oncology Group.

The antitumor effect of thalidomide is attributed to the inhibition of tumor angiogenic activity, induction of apoptosis in vitro and reduction of the high levels of angiogenic factors, such as vascular endothelial growth factor and basic fibroblast growth factor in patients with prostate cancer (6-8). Thalidomide was primarily assessed by a decline in PSA expression at doses of 100-200 mg/day (8). A phase 2 study reported that low-dose thalidomide (100 mg daily for $\leq 6$ months) was effective in reducing PSA levels: $37.5 \%$ of the treated patients exhibited a median decrease in PSA levels of $48 \%$, whereas a decrease of $50 \%$ was sustained throughout treatment for $15 \%$ of the patients. Another phase 2 trial compared low-dose (200 mg/day) and high-dose ( $\leq 1,200 \mathrm{mg}$ /day) thalidomide in patients with metastatic androgen-independent prostate cancer (AIPC). Serum PSA decreases of $50 \%$ were observed in $18 \%$ of the patients in the low-dose arm. Patients in the high-dose arm exhibited no PSA decrease, although the side effects limited dose escalation to $>200 \mathrm{mg} / \mathrm{day}$ in $30 \%$ of the cases. A total of $27 \%$ of the patients exhibited a PSA decrease of $40 \%$, often associated with improvement of the clinical symptoms $(8,9)$.

Prostate cancer cells kill mature dendritic cells (DCs), inhibit DC proliferation, differentiation and maturation, thereby reducing the number of DCs and inhibiting their function. DC deficiency and dysfunction is a major cause of prostate cancer immune evasion. Small et al (10) demonstrated the biological activity of GM-CSF (PSA decrement) in a series of androgen-independent and hormonal therapy-naive prostate cancer patients. Rini et al $(11,12)$ investigated the biological effect of GM-CSF $\left(250 \mu \mathrm{g} / \mathrm{m}^{2} /\right.$ day $)$, as measured by PSA kinetics, in 30 patients with increasing PSA levels following radical prostatectomy or radiotherapy. Of the 29 evaluable patients, $3(10 \%)$ achieved a 50\% reduction in PSA [95\% confidence interval (CI): 2-27\%]. In the 26 patients whose pre-treatment PSA doubling time (PSADT) was calculated, the median PSADT increased from 8.4 to 15 months $(\mathrm{P}<0.001)$ and the median slope of the PSA vs. time curve decreased with treatment $(\mathrm{P}<0.004)$. Of the 29 evaluable patients, $7(24 \%)$ remained free of disease progression after a median of 4.4 years (range, 4.0-4.8 years) of GM-CSF treatment.

Dreicer et al (5) conducted a phase 2 trial of thalidomide plus GM-CSF in 22 patients with metastatic AIPC. GM-CSF $(250 \mu \mathrm{g})$ was administered 3 times per week, with thalidomide titrated to $200 \mathrm{mg} / \mathrm{day}$, for 6 months. All 22 patients exhibited 
Table II. Prostate-specific antigen (PSA) levels and percentage decrease in cycle 1.

\begin{tabular}{lcrrrrrrrrrrr}
\hline & \multicolumn{10}{c}{ Patients } \\
\cline { 2 - 12 } PSA $(\mu \mathrm{g} / \mathrm{l})$ & 1 & 2 & 3 & 4 & 5 & 6 & 7 & 8 & 9 & 10 & 11 \\
\hline Day 1 & 95.09 & 98.32 & $4,667.1$ & 494.8 & 32.07 & 47.08 & 19.31 & 11.04 & 11.72 & 28.80 & 511.10 \\
Day 8 & 34.74 & 91.03 & 3,878 & 488.9 & 16.75 & 24.21 & 14.46 & 2.71 & 10.88 & 13.75 & 295.50 \\
Decrease $(\%)$ & 63.50 & 7.40 & 16.90 & 1.2 & 47.80 & 48.60 & 25.10 & 75.50 & 7.20 & 52.40 & 42.20 \\
\hline
\end{tabular}

Table III. Prostate-specific antigen (PSA) levels and percentage decrease in cycle 2.

\begin{tabular}{lccccccccccr}
\hline & \multicolumn{10}{c}{ Patients } \\
\cline { 2 - 12 } PSA $(\mu \mathrm{g} / \mathrm{l})$ & \multicolumn{1}{c}{1} & \multicolumn{1}{c}{2} & \multicolumn{1}{c}{3} & \multicolumn{1}{c}{4} & \multicolumn{1}{c}{5} & \multicolumn{1}{c}{6} & 7 & 8 & 9 & 10 & 11 \\
\hline Day 1 & 19.81 & 56.29 & 3,378 & 494.1 & 15.78 & 16.02 & 9.34 & 1.12 & 7.46 & 6.93 & 152.3 \\
Day 8 & 30.91 & 54.26 & 2,138 & 449.7 & 18.03 & 19.83 & 9.15 & 0.79 & 7.39 & 6.48 & 136.4 \\
Decrease (\%) & -56.00 & 3.60 & 36.7 & 9.0 & -14.30 & -23.80 & 2.00 & 29.50 & 0.90 & 6.50 & 10.4 \\
\hline
\end{tabular}

Table IV. Prostate-specific antigen (PSA) levels and percentage decrease in cycle 3.

\begin{tabular}{lrrrrrrrrrrr}
\hline & \multicolumn{10}{c}{ Patients } \\
\cline { 2 - 12 } PSA $(\mu \mathrm{g} / \mathrm{l})$ & \multicolumn{1}{c}{1} & \multicolumn{1}{c}{2} & \multicolumn{1}{c}{3} & \multicolumn{1}{c}{4} & \multicolumn{1}{c}{5} & \multicolumn{1}{c}{6} & 7 & 8 & 9 & 10 & 11 \\
\hline Day 1 & 13.90 & 52.25 & 2,118 & 295.7 & 14.30 & 7.80 & 6.71 & 0.36 & 3.87 & 3.20 & 126.40 \\
Day 8 & 9.19 & 35.95 & 1,613 & 317.3 & 10.69 & 5.29 & 6.09 & 0.13 & 2.08 & 1.38 & 80.94 \\
Decrease (\%) & 33.90 & 31.20 & 23.8 & -7.3 & 25.20 & 32.20 & 9.20 & 63.90 & 46.30 & 56.90 & 36.00 \\
\hline
\end{tabular}

Table V. Prostate-specific antigen (PSA) levels and percentage decrease in cycle 4.

\begin{tabular}{lrrrrrrrrrrr}
\hline & \multicolumn{10}{c}{ Patients } \\
\cline { 2 - 12 } PSA $(\mu \mathrm{g} / \mathrm{l})$ & \multicolumn{1}{c}{1} & \multicolumn{1}{c}{2} & \multicolumn{1}{c}{3} & \multicolumn{1}{c}{4} & \multicolumn{1}{c}{5} & \multicolumn{1}{c}{6} & \multicolumn{1}{c}{7} & \multicolumn{1}{c}{8} & \multicolumn{1}{c}{9} & 10 & 11 \\
\hline Day 1 & 6.04 & 43.69 & 832.6 & 286.2 & 6.01 & 3.02 & 5.47 & 0.11 & 1.55 & 1.11 & 38.64 \\
Day 8 & 4.11 & 31.30 & 705.4 & 224.1 & 4.32 & 1.62 & 3.87 & 0.21 & 0.91 & 1.68 & 31.84 \\
Decrease $(\%)$ & 32.00 & 28.40 & 15.3 & 21.7 & 28.10 & 46.40 & 29.30 & -90.90 & 42.30 & -51.40 & 18.00 \\
\hline
\end{tabular}

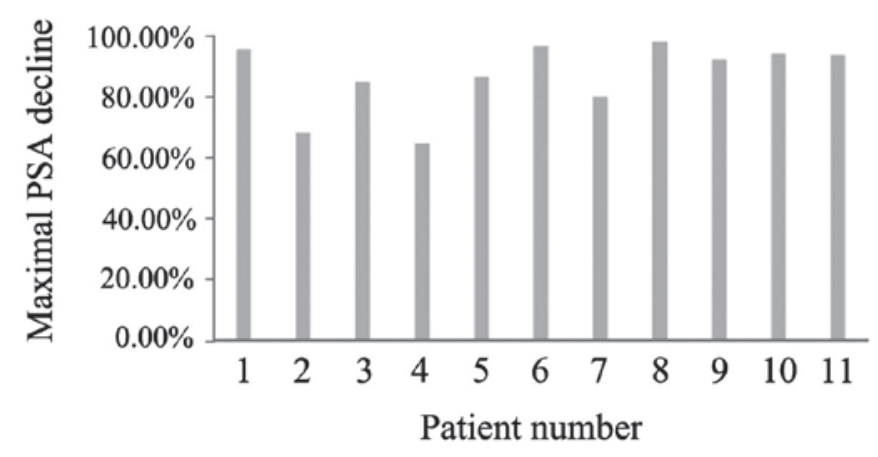

Figure 1. PSA response (maximal percentage decrease) in the 11 study patients. PSA, prostate-specific antigen.
Table VI. Toxicities associated with granulocyte-macrophage colony-stimulating factor plus thalidomide in patients with castration-resistant prostate cancer.

\begin{tabular}{lccc}
\hline Toxicities & Grade 1 & Grade 2 & Grade 3 \\
\hline Fever & 4 & 0 & 0 \\
Nausea & 4 & 0 & 0 \\
Tinnitus & 1 & 0 & 0 \\
Diarrhea & 1 & 0 & 0 \\
Constipation & 7 & 0 & 0 \\
Neuropathy & 2 & 1 & 0 \\
Fatigue & 2 & 1 & 0 \\
Skin rash & 0 & 1 & 0 \\
Deep venous thrombosis & 1 & 0 & 0 \\
\hline
\end{tabular}


decreased PSA levels 2 weeks after treatment. A total of 5 patients exhibited a $50 \%$ decrease in PSA from baseline (median, $58 \mathrm{ng} / \mathrm{ml}$ ), verified at 4 weeks after best response. The observed response rate was 23\% (95\% CI: 8-45\%).

Amato et al (13) performed another phase 2 trial of thalidomide plus GM-CSF in 21 patients with hormone-naïve prostate cancer. GM-CSF $\left(250 \mu \mathrm{g} / \mathrm{m}^{2}\right.$; maximum, $\left.500 \mu \mathrm{g}\right)$ was administered 3 times per week, with thalidomide titrated to $100 \mathrm{mg}$ /day (maximum, $400 \mathrm{mg}$ ). In the 18 patients who responded to this treatment, the median PSA reduction was $59 \%$ (range, 26-89\%) and 12 patients (67\%) exhibited a reduction of $\geq 50 \%$.

In our study, the median age of the 11 patients was 77 years and, as determined by the NCCN guidelines, the patients were not suitable for chemotherapy with taxane-based regimens. In addition, abiraterone and sipuleucel-T have not been approved for use in China. After obtaining permission from the patients and their families, GM-CSF plus thalidomide was selected as the treatment regimen. The results indicated that this combination was clinically effective in older (aged $\geq 70$ years, with a life expectancy of $>1$ year) patients with CRPC (Fig. 1). In our study, 3 patients $(27.2 \%)$ had a PSA rebound, with 1 patient exhibiting a PSA rebound of $>50 \%$ (Table III) in cycle 2 ; 1 patient $(27.2 \%$ ) had a PSA rebound of $<50 \%$ (Table IV) in cycle 3 ; and 2 patients (18.2\%) had PSA rebounds of $>50 \%$ (Table V) in cycle 4. PSA rebound was attributed to extensive tumor cell necrosis caused by the treatment, which may exhibit significant individual differences; therefore, the changing trend of PSA during treatment should be the main focus rather than the PSA level. In responders, the overall median decrease in PSA was $92.2 \%$, although several patients exhibited a PSA rebound in different cycles. The regimen was well tolerated, with mild (grade 1-2) toxicities. The most frequent grade 1-2 toxicities were fever, nausea, constipation, neuropathy and fatigue, whereas 1 patient developed grade 1 deep venous thrombosis.

In conclusion, our study demonstrated that the combination of GM-CSF plus thalidomide was clinically effective and well tolerated and may be considered an optimal treatment choice for elderly patients with CRPC.

\section{References}

1. Loblaw DA, Virgo KS, Nam R, et al: Initial hormonal management of androgen-sensitive metastatic, recurrent, or progressive prostate cancer. J Clin Oncol 25: 1596-1605, 2007.

2. Tannock IF, de Wit R, Berry WR, et al: Docetaxel plus prednisone or mitoxantrone plus prednisone for advanced prostate cancer. N Engl J Med 351: 1502-1512, 2004.

3. Bissery MC, Vrignaud P and Bayssas M: Preclinical in vivo activity of docetaxel containing combinations. Proc Am Soc Clin Oncol 14: 489, 1995

4. Kantoff PW, Higano CS, Shore ND, et al: Sipuleucel-T immunotherapy for castration-resistant prostate cancer. N Engl J Med 363: 411-422, 2010.

5. Dreicer R, Klein EA, Elson P, et al: Phase II trial of GM-CSF thalidomide in patients with androgen-independent metastatic prostate cancer. Urol Oncol 23: 82-86, 2005.

6. Franks ME, Macpherson GR and Figg WD: Thalidomide. Lancet 363: 1802-1811, 2004.

7. D'Amato RJ, Loughnan MS, Flynn E, et al: Thalidomide is an inhibitor of angiogenesis. Proc Natl Acad Sci USA 91: 4082-4085, 1994.

8. Drake MJ, Robson W, Mehta P, et al: An open-label phase II study of low-dose thalidomide in androgen-independent prostate cancer. Br J Cancer 88: 822-827, 2003.

9. Figg WD, Dahut W, Duray P, et al: A randomized phase II trial of thalidomide, an angiogenesis inhibitor, in patients with androgen-independent prostate cancer. Clin Cancer Res 7: 1888-1893, 2001.

10. Small EJ, Reese DM, Um B, et al: Therapy of advanced prostate cancer with granulocyte macrophage colony-stimulating factor. Clin Can Res 5: 1738-1744, 1999.

11. Rini B, Weinberg V, Bok R and Small EJ: Prostate-specific antigen kinetics as a measure of the biologic effect of granulocyte-macrophage colony-stimulating factor in patients with serologic progression of prostate cancer. J Clin Oncol 21: 99-105, 2003.

12. Rini BI, Fong L, Weinberg V, Kavanaugh B and Small EJ: Clinical and immunological characteristics of patients with serologic progression of prostate cancer achieving long-term disease control with granulocyte-macrophage colony-stimulating factor. J Urol 75: 2087-2091, 2006.

13. Amato RJ, Hernandez-McClain J and Henary H: Phase 2 study of granulocyte-macrophage colony-stimulating factor plus thalidomide in patients with hormone-naïve adenocarcinoma of the prostate. Urol Oncol 27: 8-13, 2009. 